\title{
Identification of the Higgs boson produced in association with top quark pairs in proton-proton collision: an analysis of the final state containing three leptons with the ATLAS detector
}

\author{
Valentina Vecchio, on behalf of the ATLAS Collaboration \\ Università e INFN Roma Tre (IT) \\ E-mail: valentina.vecchiodcern.ch
}

\begin{abstract}
The associated production of the Higgs boson with top quarks $(t \bar{t} H)$ allows to constrain the top Yukawa coupling. This coupling is a key parameter of the Standard Model and its direct study through the $t \bar{t} H$ production mode is one of the most challenging in the ATLAS physics program at LHC. An explorative analysis for this process has been performed using $13.2 \mathrm{fb}^{-1}$ of Run-2 data recorded by the ATLAS detector in 2015 and 2016 at a center of mass energy of $13 \mathrm{TeV}$. The studied final state has three charged light leptons (electrons and muons) and hadronic jets. The primary targets are the Higgs boson decays in vector bosons and tau leptons $(H \rightarrow W W, H \rightarrow Z Z$, $H \rightarrow \tau \tau)$. This paper describes the physical objects definition, the analysis strategy, the estimation and the modeling of the main backgrounds. Results are shown in terms of the ratio between the measured cross section and the one predicted by the Standard Model.
\end{abstract}

EPS-HEP 2017, European Physical Society conference on High Energy Physics 5-12 July 2017

Venice, Italy 


\section{Introduction}

The interaction of the Higgs boson [1] [2] with fermions is described by a Yukawa term in the Standard Model (SM) Lagrangian. The expression for the coupling strength of the Yukawa interaction is the following:

$$
y_{f}=\frac{m_{f} \times \sqrt{2}}{v}
$$

where $m_{f}$ is the mass of the fermion and $v$ the vacuum expectation value $(\approx 246 \mathrm{GeV})$, which gives the energy scale of the Electro Weak Symmetry Breaking (EWSB).

Given the direct measurement of the top-quark mass $m_{t}=173.21 \pm 0.51 \pm 0.71 \mathrm{GeV}$ [3], the topquark Yukawa coupling is $\approx 1$. This value is the largest one in the SM and suggests that the coupling on the top quark to the Higgs boson could play a special role in the EW context. The associated production of the Higgs boson with a quark top pair $(t \bar{t} H)$ allows to measure directly this Yukawa coupling. The $t \bar{t} H$ process has the smallest cross section if compared to the other Higgs production processes ( $\mathrm{ggH}, \mathrm{VBF}$ and $\mathrm{VH})$ and it has not been yet observed.

Indirect constraints on the top-quark Yukawa coupling can be inferred from gluon-gluon fusion production of the Higgs boson, as well as from $H \rightarrow \gamma \gamma$ decays, since the heaviest SM particles are expected to provide the dominant contributions to the loops in both production and decay. However, this assumes that no additional, yet unknown, heavy particles coupling to the Higgs boson enter these loops. It is therefore of the highest importance to measure such coupling directly from $t \bar{t} H$. The ATLAS experiment searches for the $t \bar{t} H$ process in different finale states, categorized according to the Higgs boson decays:

- $t \bar{t} H \rightarrow t \bar{t}+\gamma \gamma$

- $t \bar{t} H \rightarrow t \bar{t}+b \bar{b}$

- $t \bar{t} H \rightarrow t \bar{t}+W W^{*} / Z Z^{*} / \tau \tau$

The latter one is commonly referred to as multilepton final state and is divided into sub-channel as a function of the number of prompt leptons: from two up to four. The three lepton final state $t \bar{t} H \rightarrow 3 \ell+X$ is mostly sensitive to $H \rightarrow W W^{*}$ (74\% from simulation assuming SM branching fractions); its analysis is described in this contribution. The measurement has been performed using $13.2 \mathrm{fb}^{-1}$ of data collected by the ATLAS detector and produced in $\sqrt{s}=13 \mathrm{TeV}$ protonproton collisions delivered by the Large Hadron Collider (LHC)

\section{Event selection}

For the sake of simplicity the experimental setup and the reconstruction of physical object is not reported. The ATLAS experiment is described in detail in Ref. [4]. Physical objects definition are listed together with their reconstruction performance references: vertex [5], electrons [6], muons [7], jets [8] [9], b-tagged jets [10] [11], hadronically decaying tau leptons [12], missing transverse momentum [13].

Selected events are required to have at least one vertex reconstructed and the primary vertex is chosen to be the reconstructed vertex with the largest $\Sigma p_{T}^{2}$ from all the associated tracks. A single lepton trigger is applied. Three prompt light leptons $(e / \mu)$ are selected and classified as follows: 
- $\ell_{0}$ is the lepton with the opposite charge with respect to the other two

- $\ell_{1}$ is the closest lepton in $\Delta R^{1}$ to the $\ell_{0}$

- $\ell_{2}$ is the remaining one

A threshold in $p_{T}$, together with selections on the isolation and on variables sensitive to the association to the vertex, are applied to leptons. Concerning the requirement on the number of jets two case are allowed:

- exactly three jets of which at least two b-tagged

- at least four jets of which one b-tagged

Finally, different vetoes on the invariant mass of leptons are applied: a low mass veto and a $\mathrm{Z}$ mass veto. The latter one aims to reduce the contribution from the production of resonant $\mathrm{Z}$ boson in association with top quark pairs $(t \bar{t} Z)$.

\section{Backgrounds}

Backgrounds in the signal region described above are categorized into those in which all the selected leptons are produced in decays of electroweak bosons or $\tau$ leptons (prompt leptons) and those in which at least one lepton arises from another source. In the latter case, the leptons arise from hadron decays or photon conversions (non-prompt), other interactions in detector material (charge mis-reconstruction or fake), or improper reconstruction of other particle species (fake).

\subsection{Background sources with prompt leptons}

These backgrounds are estimated using Monte Carlo (MC) simulation. In the studied final state, the main contribution with prompt leptons comes from the production of vector bosons in association with top-quark pairs $t \bar{t} V(V=Z / \gamma / W)$. Diboson production, in particular WZ, in association with heavy quarks is the subleading contribution. The largest backgrounds with prompt leptons are studied in validation regions (VR) that are disjoint from the signal region and enhance specific processes. The validation regions are described in Table 3.1. No high-yield validation region for $t \bar{t} W$ is available. A total uncertainty of $56 \%$ is associated to its cross-section extracted from a preliminary measurement of this process at $\sqrt{s}=13 \mathrm{TeV}$ [14]. Figures 1 and 2 show the data and simulation distribution in two different validation regions.

\subsection{Background sources with non-prompt and fake leptons}

Backgrounds involving non-prompt and fake leptons are estimated from data using a transfer factor from leptons that are required explicitly to fail the analysis requirements. The major process that in this case is the dileptonic decay of $t \bar{t}$, the cross-section of which is one of the highest at LHC ${ }^{2}$. The control region in which the transfer factor is evaluated is defined reversing the isolation requirement on $\ell_{1}$ or $\ell_{2}$. The systematic uncertainty on the transfer factor arises from the extrapolation from the low jet multiplicity region to the signal region. Another source is the statistical uncertainty in the data yield in the control region.

\footnotetext{
${ }^{1} R=\sqrt{\phi^{2}+\eta^{2}}$

${ }^{2} \sigma(t \bar{t} \rightarrow \ell v \ell v b \bar{b}) \approx 70 \mathrm{pb}, \sigma(t \bar{t} H \rightarrow 3 \ell+X) \approx 0.50 \mathrm{fb}$
} 
Table 1: Selections for the validation regions of the main backgrounds with prompt leptons

\begin{tabular}{ll}
\hline \hline Process & Selection criteria \\
\hline Tight $t \bar{t} Z$ & At least one $\ell^{+} \ell^{-}$pair with $\operatorname{lm}\left(\ell^{+} \ell^{-}\right)-91.2 \mathrm{GeVK}<10 \mathrm{GeV}$ \\
& $N_{\text {jets }} \geq 4$ and $N_{b-\text { jets }} \geq 2$ \\
\hline Loose $t \bar{t} Z$ & At least one $\ell^{+} \ell^{-}$pair with $\operatorname{lm}\left(\ell^{+} \ell^{-}\right)-91.2 \mathrm{GeVK}<10 \mathrm{GeV}$ \\
& $N_{\text {jets }} \geq 1$ and $N_{b-\text { jets }} \geq 1$, or $N_{\text {jets }}=3$ and $N_{b-\text { jets }} \geq 2$ \\
\hline WZ+1b-tag & At least one $\ell^{+} \ell^{-}$pair with $\operatorname{lm}\left(\ell^{+} \ell^{-}\right)-91.2 \mathrm{GeVK} 10 \mathrm{GeV}$ \\
& $N_{\text {jets }} \geq 1$ and $N_{b-\text { jets }}=1$ \\
\hline \hline
\end{tabular}

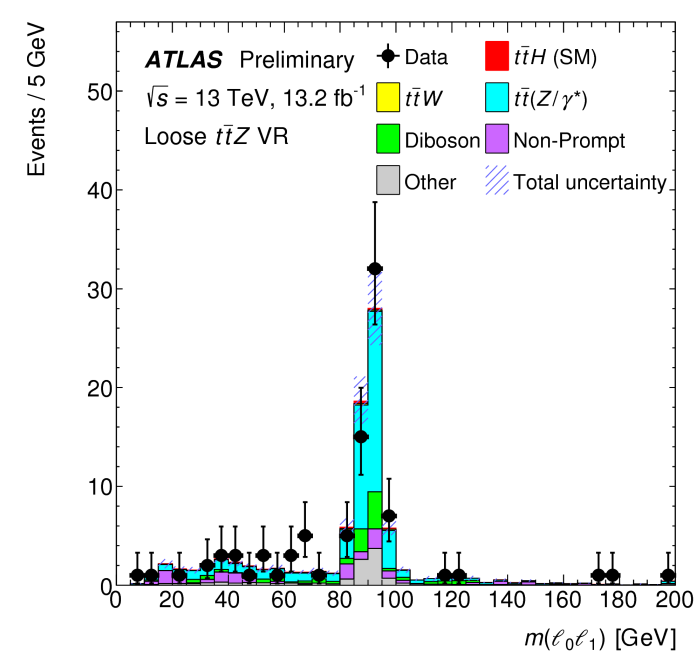

Figure 1: Comparison between data and simulation in the Loose $t \bar{t} Z$ validation region [15]

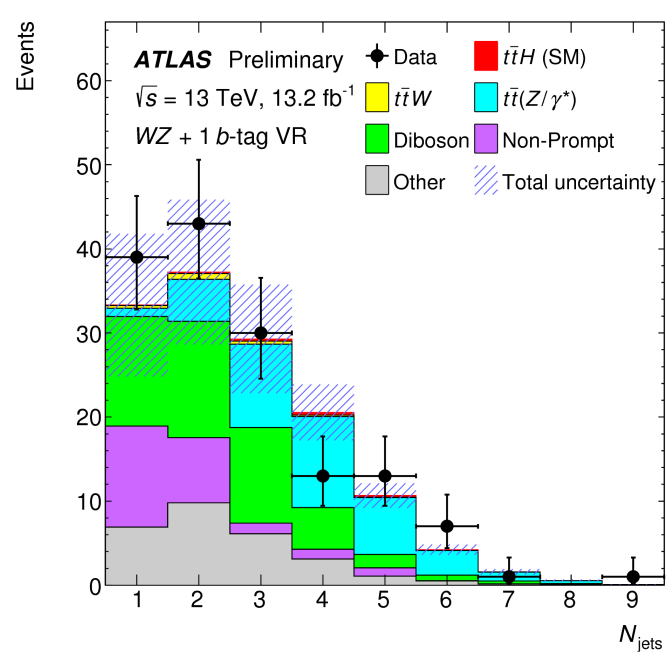

Figure 2: Comparison between data and simulation in the WZ+1b-tag validation region [15]

\section{Systematic uncertainties}

The systematic uncertainties arise from theoretical predictions and detector related effects. The uncertainty on non-prompt estimates have the largest effects on the background estimates.

The most important uncertainty arising from the theoretical predictions is the one on the modeling of the acceptance for $t \bar{t} W$ events with high jet multiplicity. Systematic uncertainties on the acceptance for the $t \bar{t} V$ backgrounds are derived using MC event simulation with several theoretical (QCD) parameter values.

The most important detector-related systematic uncertainty arises from the efficiency of the jet-tovertex association method of approximately $2.5 \%$ per jet with $p_{T}<60 \mathrm{GeV}$. The uncertainty in the modeling of pileup interactions and the jet energy scale also contribute significantly.

A detailed list of all the contributions is reported in Table 4. Here their effect on the uncertainty of the signal strength $\mu$ is reported, where $\mu$ is defined as the ratio between the measured cross section and the one predicted by the SM. The resulting signal strength is the one for the combination of all the multilepton channel. 
Table 2: Summary of the effects of the systematic uncertainties on the signal strength.

\begin{tabular}{lc}
\hline \hline Uncertainty Source & $\Delta \mu$ \\
\hline Non-prompt leptons and charge mis-reconstruction & $+0.56-0.64$ \\
Jet-vertex association, pileup modeling & $+0.48-0.36$ \\
$t \bar{t} W$ modeling & $+0.29-0.31$ \\
$t \bar{t} H$ modeling & $+0.31-0.15$ \\
Jet energy scale and resolution & $+0.22-0.18$ \\
$t \bar{t} Z$ modeling & $+0.19-0.19$ \\
Luminosity & $+0.19-0.15$ \\
Diboson modeling & $+0.15-0.14$ \\
Jet flavor tagging & $+0.15-0.12$ \\
Light lepton $e \mu$ and $\tau_{\text {had }}$ ID, isolation, trigger & $+0.12-0.10$ \\
Other background modeling & $+0.11-0.11$ \\
\hline Total systematic uncertainty & $+1.1-0.9$ \\
\hline \hline
\end{tabular}

\section{Results}

The best-fit value of the $t \bar{t} H$ signal strength is obtained using a maximum likelihood fit to the data yields of all the categories. Since no observation of the signal is obtained, the likelihood function is also used to obtain 95\% confidence level (CL) upper limit of 4.9 (2.3 expected). Results are shown in Figure 3 and 4.

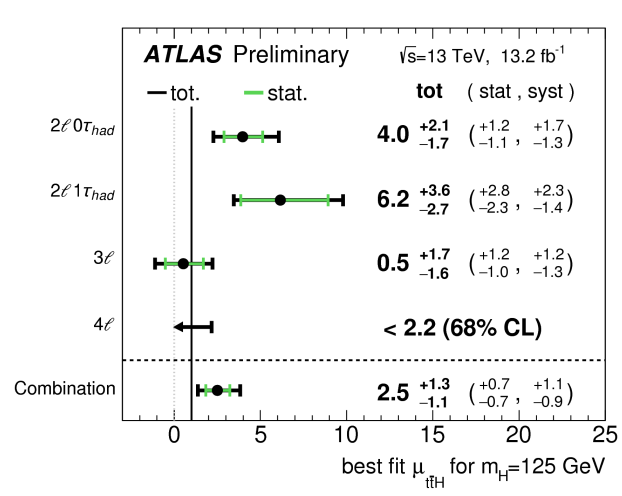

Figure 3: Best-fit value of the signal strength [15] for Figure 4: Comparison between data and simulation each multilepton channel and their combination.



in $3 \ell$ signal region [15]

\section{References}

[1] P. W. Higgs, "Broken Symmetries And The Masses Of Gauge Bosons", Phys. Rev. Lett. 13 (1964)

[2] F. Englert and R. Brout, "Broken Symmetry and the Mass of Gauge Vector Mesons", Phys. Rev. Lett. 13 (1964)

[3] C. Patrignani et al. (Particle Data Group), "Review of Particle Physics", Chin. Phys. C, 40, 100001 (2016)

[4] ATLAS Collaboration, "The ATLAS Experiment at the CERN Large Hadron Collider", JINST 3 (2008) S08003 
[5] ATLAS Collaboration, "Vertex Reconstruction Performance of the ATLAS Detector at $\sqrt{s}=13 \mathrm{TeV}$ ", ATL-PHYS-PUB-2015-026, https://cds.cern.ch/record/2037717

[6] ATLAS Collaboration, "Electron efficiency measurements with the ATLAS detector using the 2015 LHC proton-proton collision data", ATLAS-CONF-2016-024, https://cds.cern.ch/record/2157687

[7] ATLAS Collaboration "Muon reconstruction performance of the ATLAS detector in proton-proton collision data at $\sqrt{s}=13 \mathrm{TeV}$ ", Eur. Phys. J. C(2016) 76:292

[8] M. Cacciari, G. P. Salam, and G. Soyez, "The anti-kt jet clustering algorithm", JHEP 04 (2008) 063

[9] ATLAS Collaboration, "Selection of jets produced in $13 \mathrm{TeV}$ proton-proton collisions with the ATLAS detector", ATLAS-CONF-2015-029, https://cds.cern.ch/record/2037702?ln=it

[10] ATLAS Collaboration "Performance of b-Jet Identification in the ATLAS Experiment", JINST 11 P04008 (2016)

[11] ATLAS Collaboration "Expected performance of the ATLAS b-tagging algorithms in Run-2", ATL-PHYS-PUB-2015-022, https://cds.cern.ch/record/2037697?ln=it

[12] ATLAS Collaboration "Reconstruction, Energy Calibration, and Identification of Hadronically Decaying Tau Leptons in the ATLAS Experiment for Run-2 of the LHC", ATL-PHYS-PUB-2015-045, https://cds.cern.ch/record/2064383?ln=it

[13] ATLAS Collaboration "Performance of missing transverse momentum reconstruction for the ATLAS detector in the first proton-proton collisions at $\sqrt{s}=13 \mathrm{TeV} "$, ATL-PHYS-PUB- 2015-027, https://cds.cern.ch/record/2037904?ln=it

[14] ATLAS Collaboration, "Measurement of the $t \bar{t} Z$ and $t \bar{t} W$ production cross sections in multilepton final states using $3.2 \mathrm{fb}^{-1}$ of pp collisions at $13 \mathrm{TeV}$ at the LHC", (2016), ATLAS-CONF-2016-003, https://cds.cern.ch/record/2138947

[15] ATLAS Collaboration, "Search for the Associated Production of a Higgs Boson ad a Top Quark Pair in Multilepton Final States with the ATLAS Detector", ATLAS-CONF-2016-058, https://cds.cern.ch/record/2206153 